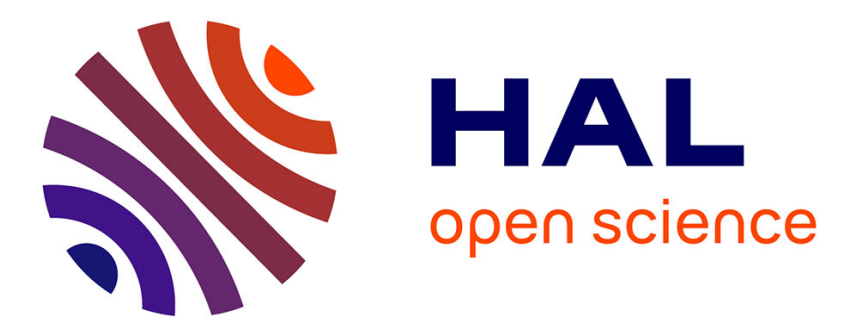

\title{
Motion estimation in 3D echocardiography using smooth field registration
}

Oudom Somphone, Cecile Dufour, Benoît Mory, Loïc Hilpert, Sherif Makram-Ebeid, Nicolas Villain, Mathieu de Craene, Allain Pascal, Eric Saloux

\section{- To cite this version:}

Oudom Somphone, Cecile Dufour, Benoît Mory, Loïc Hilpert, Sherif Makram-Ebeid, et al.. Motion estimation in 3D echocardiography using smooth field registration. Third International Workshop, STACOM 2012, Held in Conjunction with MICCAI 2012, Oct 2012, Nice, France. pp.151-158, 10.1007/978-3-642-36961-2_18. hal-00924983

\section{HAL Id: hal-00924983 \\ https://hal.science/hal-00924983}

Submitted on 7 Jan 2014

HAL is a multi-disciplinary open access archive for the deposit and dissemination of scientific research documents, whether they are published or not. The documents may come from teaching and research institutions in France or abroad, or from public or private research centers.
L'archive ouverte pluridisciplinaire HAL, est destinée au dépôt et à la diffusion de documents scientifiques de niveau recherche, publiés ou non, émanant des établissements d'enseignement et de recherche français ou étrangers, des laboratoires publics ou privés. 


\title{
Motion Estimation in 3D Echocardiography using Smooth Field Registration
}

\author{
Oudom Somphone ${ }^{1}$, Cécile Dufour ${ }^{1}$, Benoît Mory ${ }^{1}$, Loïc Hilpert ${ }^{2}$, \\ Sherif Makram-Ebeid ${ }^{1}$, Nicolas Villain ${ }^{1}$, Mathieu De Craene ${ }^{1}$, Pascal Allain ${ }^{1}$, \\ and Eric Saloux ${ }^{2}$ \\ ${ }^{1}$ Medisys, Philips Research, Suresnes, France \\ ${ }^{2}$ Department of Cardiology, University Hospital of Caen, Caen, France.
}

\begin{abstract}
This paper describes an algorithm for motion and deformation quantification of $3 \mathrm{D}$ cardiac ultrasound sequences. The algorithm is based on the assumption that the deformation field is smooth inside the myocardium. Thus, we assume that the displacement field can be represented as the convolution of an unknown field with a Gaussian kernel. We apply our algorithm to datasets with reliable ground truth: a set of synthetic sequences with known trajectories and a set of sequences of a mechanical phantom implanted with microsonometry crystals.
\end{abstract}

Keywords: Motion Estimation, Myocardial Motion, 3D Cardiac Ultrasound, Demons, Phantom, Microsonometry.

\section{Introduction}

Quantifying heart function at a local scale is an important challenge for the diagnosis of heart diseases. For example, locally ischemic segments will have impaired contractility in a region whose size and location depend on vascularization territories from the coronary tree. Another example is local assynchrony induced by conduction defects in the Purkinje tree. Besides the importance of quantifying heart function at a local level, deformation (strain) is preferred to motion since it is less influenced by surrounding tissue.

Several imaging modalities offer the possibility to quantify strain noninvasively. Tissue Doppler Imaging (TDI) [1] measures tissue velocities (hence strain rate) in a $2 \mathrm{D}$ plane. However, they give a projection of the strain tensor in the direction of the ultrasound beam. Speckle tracking [2] was proposed as an alternative to quantify displacement independently from the acquisition angle. Because of the orientation of the fibers architecture within the ventricular wall [3], motion and deformation of myocardial tissue are intrinsically three dimensional. 2D speckle tracking, besides the disadvantage of providing strain information in a single plane, is therefore limited by out of plane motion, disturbing the tracking of speckle over time.

3D speckle tracking therefore has the potential to overcome limitations of both TDI and 2D speckle tracking. However, thorough validation needs to be 
conducted for quantifying the impact of different imaging settings on accuracy and localizability of this technique. Byram et al. [4] studied the effects of frame rate, kernel size, and data type on 3D tracking performances. They reported increasing accuracy in motion accuracy with frame rate, up to $200 \mathrm{~Hz}$, at which the accuracy stabilizes. Heyde et al. [5] quantified strain accuracy for a nonrigid registration method on a thick-walled cardiac phantom. Local inclusions were placed for various stiffness ratios between materials mimicking normal and pathological tissues. They reported to recover full transmural inclusions down to $17 \mathrm{~mm}$ in diameter, for a stiffness ration of at least 5:2.

In this paper, we quantify the accuracy on motion and strain of a demonsbased image registration algorithm. Motion accuracy was quantified on simulated ultrasound data for different contractility and pacing settings. Strain accuracy was quantified from in vitro phantom images by comparing relative length changes to distances measured by sonomicrometry. All data was acquired in the context of the cardiac motion analysis challenge at STACOM 2012.

\section{Motion estimation with Smooth Registration}

In this section, we give a brief description of the Smooth Registration approach that we use to perform motion estimation, and the subsequent demons-like algorithm. A Demons-based algorithm has been previously used by Mansi et al to estimate myocardial strain from 3D cine and tagged MRI sequences [6].

\subsection{Variational formulation}

Considering the problem of registering two images $R$ and $T$, referred to as the reference and the template respectively, we adopt a fluid-like regularization, which can be approximated by Gaussian linear filtering, as in the well-known demons algorithm [7]. The displacement field $\mathbf{u}$ that transforms $T$ towards $R$ is assumed to be the result of the component-wise convolution of an unknown non-rigid field $\mathbf{v}$ with a spatial Gaussian kernel $\omega_{\sigma}$ :

$$
\mathbf{u}(\mathbf{x})=\omega_{\sigma} * \mathbf{v}(\mathbf{x})=\int_{\Omega} \omega_{\sigma}(\mathbf{x}-\mathbf{y}) \mathbf{v}(\mathbf{y}) d \mathbf{y}
$$

where $\Omega \subset \mathbb{R}^{3}$ is the domain of definition of $\mathbf{u}$ and $\sigma$ is the scale of the kernel; the larger $\sigma$, the smoother $\mathbf{u}$. This field representation was introduced in [8] in the context of template-to-image registration. It is then injected within a classical Sum of Squared Differences formulation, consisting in the minimization of the following cost functional with respect to $\mathbf{v}$ :

$$
E(\mathbf{v})=\int_{\Omega}[R(\mathbf{x})-T(\mathbf{x}+\mathbf{u}(\mathbf{x}))]^{2} d \mathbf{x}
$$

Note that although the optimal displacement $\mathbf{u}$ warps $T$ to $R$ but represents the motion field at the time associated to $R$. 


\section{$2.2 \quad$ A simple algorithm}

The optimization is done by gradient descent; basic calculus of variations results in the following evolution equation:

$$
\frac{\partial \mathbf{v}}{\partial t}=-\nabla_{\mathbf{v}} E=-\omega_{\sigma} * \nabla_{\mathbf{u}} E
$$

where $\nabla_{\mathbf{u}} E$ is the gradient of $E$ w.r.t. $\mathbf{u}$ :

$$
\nabla_{\mathbf{u}} E(\mathbf{x})=-[R(\mathbf{x})-T(\mathbf{x}+\mathbf{u}(\mathbf{x}))] \nabla T(\mathbf{x}+\mathbf{u}(\mathbf{x}))
$$

It appears that the gradient w.r.t. $\mathbf{v}$ is simply obtained by filtering the gradient w.r.t. $\mathbf{u}$ with a Gaussian kernel. This leads to the demons-like iterative scheme detailed in Algorithm 1 below.

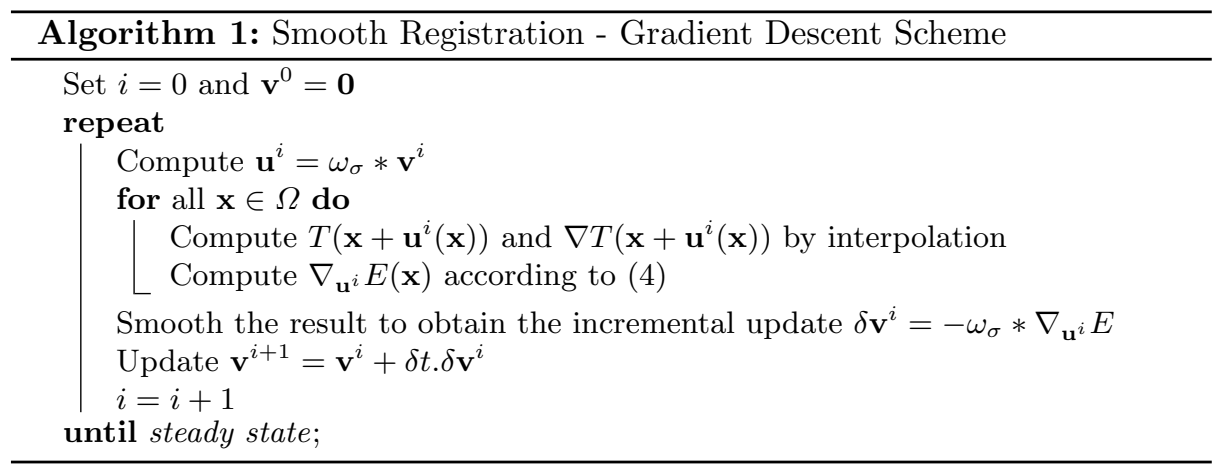

This gradient descent scheme is embedded into a multiresolution strategy.

\subsection{Myocardium mask}

Regularizing the displacement field as in (1) may corrupt the estimation inside the myocardium with the influence of features located outside of the myocardium. This is particularly critical at the interface between the pericardium - which is fixed - and the epicardium, where motion may be underestimated. To deal with this issue, we introduce a mask, provided by a prior segmentation of the myocardium, by substituting the Gaussian filtering with a normalized convolution. The displacement field becomes:

$$
\mathbf{u}=\omega_{\sigma} *(\mathbf{v}, \chi) \widehat{=} \frac{\omega_{\sigma} *(\chi \mathbf{v})}{\omega_{\sigma} * \chi}
$$

where $\chi$ is the characteristic function of the myocardium ( 1 inside and 0 outside). At voxels where $\omega_{\sigma} * \chi$ is zero, which occurs far from the mask, we set the displacement to zero. 
Replacing (1) by (5) in the previous calculus leads to the following gradient:

$$
\nabla_{\mathbf{v}} E=\chi\left(\omega_{\sigma} * \frac{\nabla_{\mathbf{u}} E}{\omega_{\sigma} * \chi}\right)
$$

The subsequent modified scheme is presented in Algorithm 2 below.

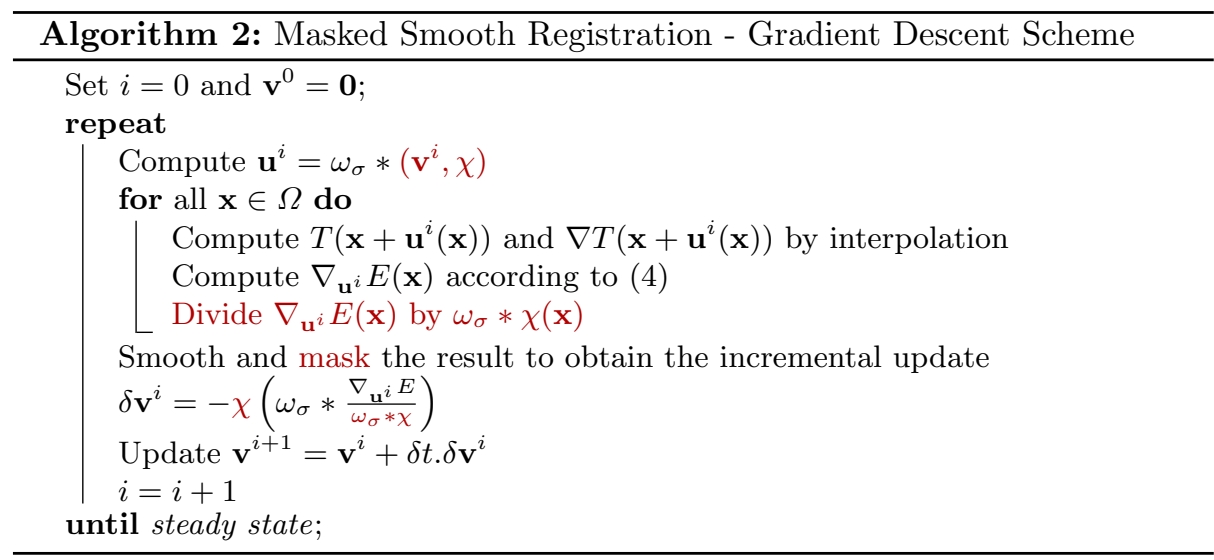

\subsection{Tracking trajectories}

Tracking trajectories along a sequence can be done by applying the above algorithm using two strategies (see Fig. 1):

(i) registering every frame $I^{(k)}$ towards the initial frame $I^{(0)}$;

(ii) registering every frame $I^{(k)}$ towards the previous frame $I^{(k-1)}$ and compose frame-to-frame displacements.

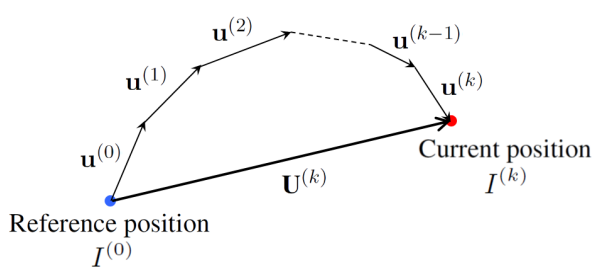

Fig. 1. Tracking trajectories along a sequence. Registering frames $I^{(k)}$ as template and $I^{(0)}$ as reference results in the displacement field $\mathbf{U}^{(k)}$. Registering frames $I^{(k)}$ as template and $I^{(k-1)}$ as reference results in the displacement field $\mathbf{u}^{(k)}$. 

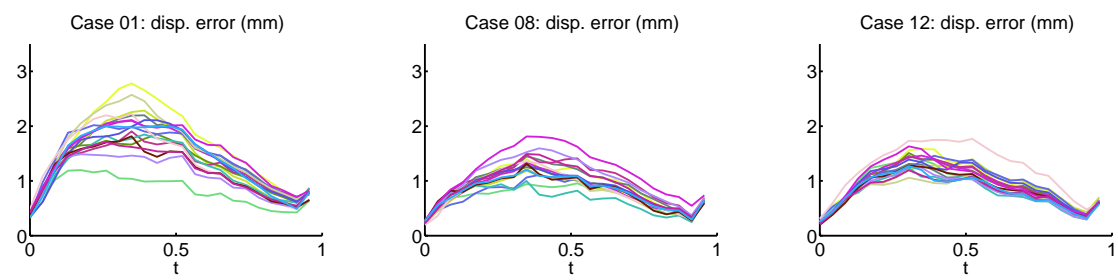

Fig. 2. Synthetic cases. Average error on displacement as a function of time for the 17 AHA segments in the 3 first cases of the database.

Strategy (i) may seem more suitable since it avoids the frame-to-frame error accumulation inherent to strategy (ii). However, it leaves out the temporal coherence of the sequence, since the estimation of each $\mathbf{U}^{(k)}$ is dependent of $I^{(0)}$ only and does not take the previous frames into account. This latter drawback being critical, we chose strategy (ii).

\section{Experiments}

We applied the method described in Sect. 2 on the synthetic and the phantom data provided for the cMAC2 challenge. Details on the data setup can be found on the cMAC2 challenge web page ${ }^{1}$. We processed all provided data i.e. the 10 simultations with provided ground truth on the motion fields and the 3 phantom datasets with ground truth provided at 4 spatial locations as distances measured by sonomicrometry. The computation time was in the order of 8 minutes for each processed sequence.

\subsection{Results on synthetic data}

On synthetic data, we evaluated the accuracy of our algorithm to estimate the trajectories of material points given on the volumetric mesh. Error magnitude was plotted over time for each of the 17 American Heart Association (AHA) segments in the Left Ventricle (LV). The curves (one per segment) are plotted in Fig. 2 for the 3 first cases of the database. Since the first case models a healthy subject, it showed the largest displacements. The error was higher on this case than for the other subjects (see Fig. 2), indicating that the error increases with the magnitude of the displacement to be estimated. The same tendency appears in the temporal evolution of the error curves within each subject. Indeed, the error increases over systole, up to frame \#10, for all cases before decreasing over diastole. The latter indicates the absence of drift in the trajectories estimated by our algorithm.

To observe the error dispersion over all subjects, we plotted the range of error magnitudes at the end of systole for each subject. The resulting plot is shown in

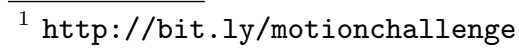




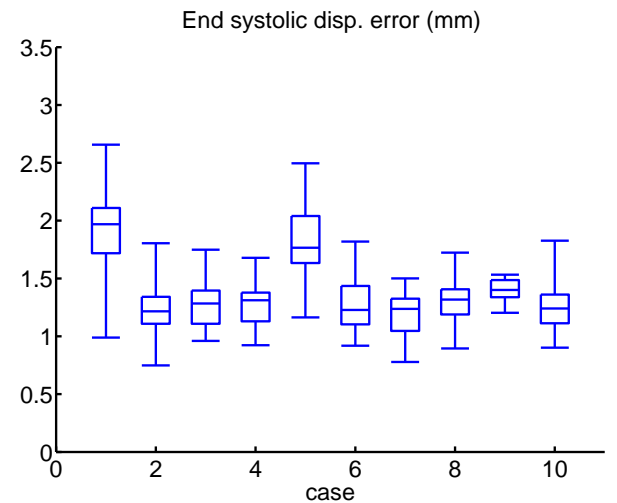

Fig. 3. Synthetic cases. Box plots showing the dispersion of displacement error (in $\mathrm{mm}$ ) measured at the end of systole for each case.

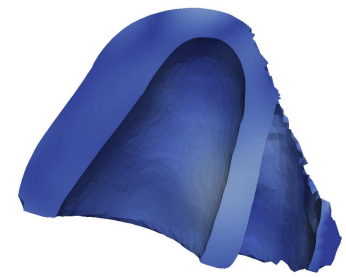

$t=0 \%$

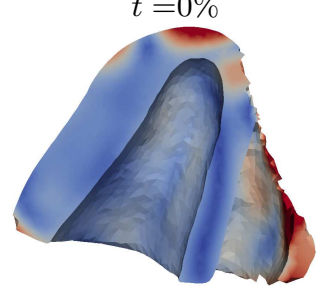

$t=55 \%$
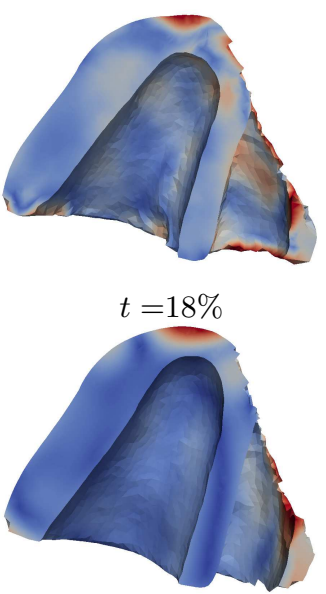

$t=73 \%$
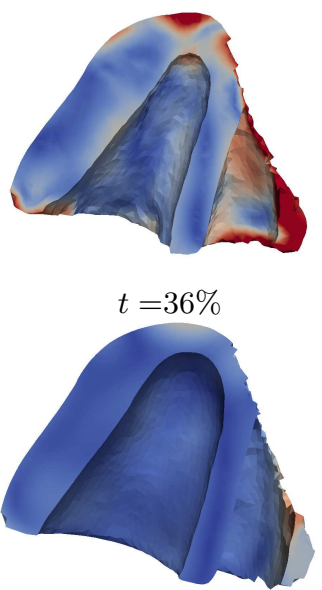

$t=91 \%$

Fig. 4. Synthetic cases. Displacement error plotted at 6 frames of the cardiac cycle (times goes from top left, early systole, to bottom right, late diastole.

Fig. 3. For most cases, the median error at the end of systole was below $1.5 \mathrm{~mm}$. Two cases exhibited higher errors: the first one, as previously commented, and the fifth case. Finally, to examine the spatial distribution of errors over time, we plotted the error on the trajectories as a color map for each frame of the cardiac cycle (see Fig. 4) for case \#1. Only the LV was tracked, which explains the high error values appearing in the right ventricle. The highest error values appeared close to the apex and can be explained by high reverberation artifacts observable on the simulated images. 


\subsection{Results on phantom data}
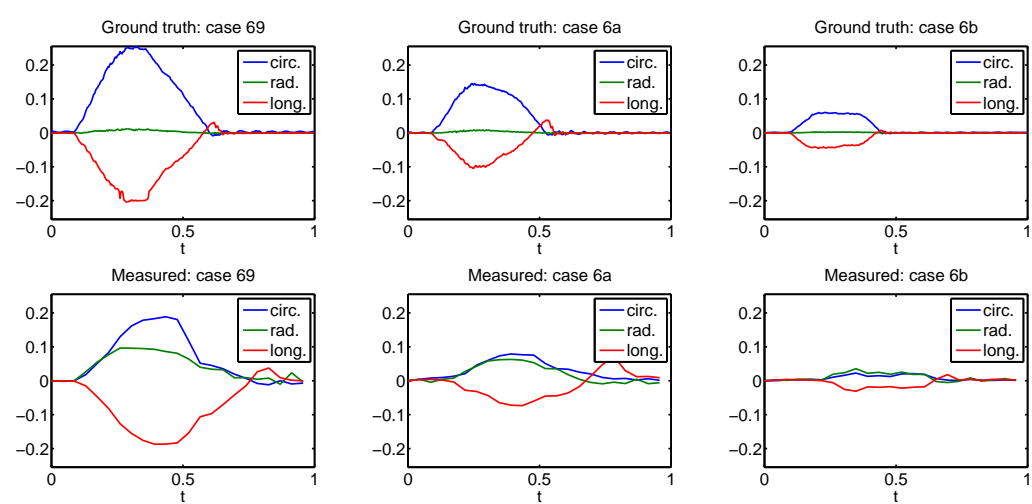

Fig. 5. Top: relative length changes over time between sonomicrometry crystals in radial, circumferential and longitudinal directions. Bottom: The same relative length changes measured by tracking on the 3D US image.

On phantom data, ground truth was provided as pairwise distances measured between sonomicrometry crystals. Crystals positions were also provided by the challenge organizers in the image space of coordinates. We computed the trajectories and the pairwise distances matching those given by the ground truth. These distances are not strictly equivalent to strain but are a good surrogate of radial, circumferential and longitudinal deformations. Fig. 5 plots on the top line the three distances as provided by sonomicrometry for each provided acquisition. It can be observed that, unlike in real myocardial tissue, the circumferencial strain takes positive values over the cardiac cycle. Radial strain showed positive values, but of one order of magnitude smaller than the two other distances. The magnitude of the global deformation was mechanically reduced over each acquisition, as confirmed by the decreasing amplitude of the sonomicrometry curves.

In comparison to the ground truth, we observe that image-based tracking (bottom line of Fig. 5) correctly estimated both the amplitude and the pattern of longitudinal strain. Only in the last acquisition, imaging noise disturbed the temporal pattern of the estimated curve with respect to ground truth. Circumferential strain was underestimated in all three acquisitions but the relative magnitude of peak-systolic circumferential strain matched the decreasing tendency of the ground truth. Finally, estimates of radial strain values are not in agreement with the values reported by the ground truth.

When averaging radial strain values on the axial slice containing the two crystals, we obtained values of respectively 10,7 and $3.5 \%$ for each acquisition. These values are significantly higher than the ones reported by sonomicrometry. 
Further experiments and validation on the estimated trajectories are required to understand this disagreement.

\section{Conclusion}

We proposed a demons-like algorithm that performs a normalized convolution of the estimated motion field in the region of interest (myocardial mask). The method is fast and processes an entire $3 \mathrm{D}$ sequence is about 7 minutes. Our experiments on the cMAC2 synthetic data gave a median accuracy around 1 $\mathrm{mm}$ at the end of systole for 8 cases over 10 . On the phantom dataset, longitudinal strain values were comparable in amplitude and pattern to distances measured by sonomicrometry. The relative amplitude and the temporal pattern of circumferential strain values were in accordance with sonomicrometry. Important differences were observed in radial strain values that require further investigation.

\section{References}

1. Sutherland, G., Di Salvo, G., Claus, P., D'hooge, J., Bijnens, B.: Strain and strain rate imaging: a new clinical approach to quantifying regional myocardial function. Journal of the American Society of Echocardiography 17(7) (2004) 788-802

2. Mondillo, S., Galderisi, M., Mele, D., Cameli, M., Lomoriello, V., Zacà, V., Ballo, P., D'Andrea, A., Muraru, D., Losi, M., et al.: Speckle-tracking echocardiography: a new technique for assessing myocardial function. Ultrasound in medicine 30(1) (2011) 71

3. Greenbaum, R., Ho, S., Gibson, D., Becker, A., Anderson, R.: Left ventricular fibre architecture in man. British heart journal 45(3) (1981) 248-263

4. Byram, B., Holley, G., Giannantonio, D., Trahey, G.: 3-d phantom and in vivo cardiac speckle tracking using a matrix array and raw echo data. Ultrasonics, Ferroelectrics and Frequency Control, IEEE Transactions on 57(4) (2010) 839-854

5. Heyde, B., Cygan, S., Choi, H., Lesniak-Plewinska, B., Barbosa, D., Elen, A., Claus, P., Loeckx, D., Kaluzynski, K., D'hooge, J.: Regional cardiac motion and strain estimation in three-dimensional echocardiography: A validation study in thick-walled univentricular phantoms. IEEE Transactions on Ultrasonics, Ferroelectrics and Frequency Control (2011)

6. Mansi, T., Pennec, X., Sermesant, M., Delingette, H., Ayache, N.: iLogDemons: A Demons-Based Registration Algorithm for Tracking Incompressible Elastic Biological Tissues. International Journal of Computer Vision 92(1) (2011) 92-111

7. Thirion, J.P.: Image matching as a diffusion process: an analogy with Maxwell's Demons. Medical Image Analysis 2(3) (1998) 243-260

8. Mory, B., Somphone, O., Prevost, R., Ardon, R.: Real-time 3D image segmentation by user-constrained template deformation. In: 15th International Conference on Medical Image Computing and Computer-Assisted Intervention (MICCAI'12). (2012) 